\title{
Philosophiques
}

\section{La femme (dite barrée) selon l’approche lacanienne}

\section{Madeleine Laroche-Parent}

Volume 12, numéro 1, printemps 1985

URI : https://id.erudit.org/iderudit/203277ar

DOI : https://doi.org/10.7202/203277ar

Aller au sommaire du numéro

Éditeur(s)

Société de philosophie du Québec

ISSN

0316-2923 (imprimé)

1492-1391 (numérique)

Découvrir la revue

Citer cet article

Laroche-Parent, M. (1985). La femme (dite barrée) selon l'approche lacanienne. Philosophiques, 12(1), 165-176. https://doi.org/10.7202/203277ar

\section{Résumé de l'article}

Selon Lacan, une femme particulière est l'objet de réduction du Pouvoir Phallique, elle l'est de par la voie sacrée et par là les femmes sont " pas-tout » dans leur désir. Il entreprend une mise en place au « Nom-du-Père » où L/a femme aura la condition de vide à la place même du jeu des substitutions. Nous tentons d'interpréter, à partir des énoncés lacaniens, ce qu'il en est de la situation féminine. d'utilisation que vous pouvez consulter en ligne.

https://apropos.erudit.org/fr/usagers/politique-dutilisation/ 


\title{
LA FEMME (DITE BARRÉE) SELON L'APPROCHE LACANIENNE
}

\author{
par Madeleine Laroche-Parent
}

\begin{abstract}
RÉSUMÉ. Selon Lacan, une femme particulière est l'objet de réduction du Pouvoir Phallique, elle l'est de par la voie sacrée et par là les femmes sont "pas-tout " dans leur désir. Il entreprend une mise en place au "Nom-du-Père » où $\mathrm{L} / \mathrm{a}$ ' femme aura la condition de vide à la place même du jeu des substitutions. Nous tentons d'interpréter, à partir des énoncés lacaniens, ce qu'il en est de la situation féminine.
\end{abstract}

ABSTRACT. In Jacques Lacan's view, a woman is a reduced object of Phallic Power through the sacred path. Women are therefore "not all" in their desire. Lacan undertakes a localisation in the "Name of Father", where wo/man is given the vacuous place in the play of substitutions. Following Lacan, we attempt at an interpretation of the feminine condition.

Enfin un homme fait, d'un lieu d'autorité, la levée du voile saintement posé sur l'asexuation d'une/de femme/s. Lacan ne le fait malheureusement pas en respectant les lois en cours dans la phonè d'autorité, pire, il ose le présenter sous forme de discours "baroque ". Aussi les pertinences présentes dans son discours s'en trouvent fortement grevées.

Il faudra de la patience à qui n'est pas initié à ce langage, mais la lecture vaut d'être faite pour qui veut s'instruire sur ce qu'il en est du ratage dans le rapport de l'Homme avec l'Autre, ici la Femme (en supposant que la lecture de ce qui suit vous donne goût à la lecture de Lacan).

Faut-il, pour bien comprendre Lacan, « replacer (son discours) dans l'histoire du plan idéologique qui le rend possible ? ». Je cite là Robert Hébert qui, lorsqu'il tente d'appréhender les fondements de la volonté de savoir, nous propose cet 
ancrage. Est-il nécessaire d'ajouter qu'il s'agit chez Lacan aussi "d'expériencè sur le terrain ${ }^{1}$ ?

Dans le développement qui suit, nous nous référons plus particulièrement au séminaire ENCORE ${ }^{2}$. Nous abordons les discours comme critique de l'utilisation d'une femme portée jusqu'à la condition mystique par un ensemble d'appareils de pouvoir, femme que Lacan dit " barrée ", qu'il écrit " L/a femme " plaçant la barre sur le LA pour souligner le fait que « de son essence elle n'est pas toute ». Le " la », dit-il, est un " article défini pour désigner l'universel $»^{3}$.

Il considère aussi cette femme forclose en un lieu où il dit ne pas y avoir de rapport sexuel possible. Cela a pour conséquence de la délier de sa propre mémoire lors même que de la chaîne de ses désirs (achevée dans la sublimation) s'instaure tout le système de renvoi de l'Ordre Phallique.

Nous délimitons notre développement à l'aide d'une proposition synthétique :

L/a femme n'est pas toute, inappropriée en son désir, ce désir - ailleurs que dans le rapport sexuel retenu - est l'objet d'un grand nia nia d'où " une femme rencontre L'homme dans la psychose " ${ }^{4}$. Ainsi le ciel qui lui échoit lui laisse jouissance "au-delà du phallus " ${ }^{5}$ alors que les chaînes de ses désirs, quoique affaire de féminité, demeurent lieu du mignon pouvoir d'où « on la dit-femme, on la diffâme $"^{6}$.

\section{"LA FEMME N'EST PAS TOUTE "}

Elle n'est pas toute là où elle n'a pas accès à tout son être, par le fait même à son essence propre. Inappropriée en son désir parce que désappropriée de celui-ci pour une grande part.

Le Désir institué ayant pour fondement le sien propre est maintenu sous forme d'abstraction pure. Ainsi est-elle forclose dans la dimension sacrée.

1. Robert Hébert, Mobiles du discours philosophique, Hurtubise HMH, 1978, p. 12-13.

2. Jacques Lacan, Le séminaire, livre XX, Encore, Seuil, 1975.

3. Encore, p. 68.

4. Jacques Lacan, Télévision, Seuil, 1973, p. 63.

5. Encore, p. 69.

6. Encore, p. 79. 
Par ailleurs, cette dimension sacrée est l'objet d'une surexploitation par divers appareils de pouvoir, entre autres celui du pouvoir " mignon ». . . lieu d'une exploitation artistique, d'où la femme ne peut sortir de la possession qu'ils exercent à son endroit, tellement elle est nouée à leur système de renvoi. Ils la laissent entrer « en emperesse pour qu'elle n'y rentre que comme signifiant m'être de cette affaire de rapport au sexe ${ }^{7}$. Lacan considère aussi que ce n'est pas dû au fait que le mignon emprunte sa mémoire désirante, qu'il y jouisse qu'elle n'est pas toute, voire que chez certaines d'entre elles se manifeste de l'hystérie lorsqu'elles " font l'homme ", mais plutôt parce que d'elle s'organise tout "l'organon" propre au pouvoir phallique ${ }^{8}$. Et que toute traduction du lieu repose sur un " phanère unique " .

Nous pouvons donc en déduire que la castration est, de fait, opérée au niveau de la dimension sacrée. L/a femme y est limitée au désir subliminal, par elle ACCOMPLI, en un système de renvoi d'ordre universel, celui des jeux d'attraits en puissance dans l'Ordre phallique. Le mignon coiffe le sacré parce qu'il assure d'une dimension universelle une créativité à laquelle il aspire $^{10}$. Ainsi la femme est doublement niée ; dans une position de forclusion (au niveau de la dimension sacrée) et anéantie au lieu des discours d'autorité (là elle est reçue qu'en vue d'une saisie de "ce qui ne se situe que du discours ») ${ }^{11}$, si bien qu'il " n'y a pas de rapport sexuel ". Lacan qualifiera tout le pouvoir « d'hommosexuel » (sic) d'où la femme se " même » au pouvoir de l'homme c'est-à-dire qu'elle se replie sur l'univers homosexuel qui la concerne (celui des femmes) ou alors elle s'accomplit " horsexe " soit sous forme purement abstraite (sans lien à l'un et l'autre des sexes tels qu'ordonnés), ainsi " être-ange ".

Nous disons qu'elle est anéantie au lieu des discours d'autorité (selon Lacan l'homme de discours exige un « une par

7. Jacques Lacan, "L'Étourdit ", revue Scilicet, no. 4, 1973, pp. 5 à 52 cit. p. 21.

8. Encore, p. $77:$ « . . . il se révèle assurément que c'est à la place, opaque, de la jouissance de l'Autre, de cet Autre en tant que pourrait l'être, si elle existait, la femme, qu'est situé cet Être suprême, mythique manifestement chez Aristote, cette sphère immobile d'où procèdent tous les mouvements, quels qu'ils soient, changements, générations, mouvements, translations, augmentations, etc.

9. "L'Étourdit", p. 21.

10. Ce que Heidegger nomme "l'être-produit ", dans Chemins, Idées/Gallimard, 1980, "L'origine de l'œuvre d'art " p. 13 à 98.

11. Encore, p. 34. 
une ${ }^{12}$, c'est qu'elles ne sont admises que comme supports de lieux d'autorité, n'étant pas accomplies au même titre qu'Une (femme) «mise au travail de l'UN » l'est ${ }^{13}$. Elles n'ont donc pas accès au système de renvoi d'autorité autrement que comme " sub-alternes ", dans cette condition elles ne risquent pas d'être sensibles aux enjeux au même titre qu'Elle.

Cette mise en place de femmes ayant fonction d'étendre le pouvoir phallique sans jamais avoir accès aux secrets qu'il supporte est comparable au montage d'un tricot à la broche nommé " point jersey "; Une maille à l'envers, une maille endroit, une autre . . . terminez le rang par Une maille à l'envers, suivi d'un rang entier Une envers. Répétez ces deux rangs jusqu'à la fin. C'est de ce rang à l'endroit que se tiennent en surface les actes d'autorité supportant monde et choses.

Retenons prioritairement cette perspective; il y a une femme retenue en tant qu'instrument, elle est l'objet de la Réduction. Ainsi lorsqu'il est dit " $\mathrm{L} / \mathrm{a}$ femme » il serait bon d'entendre aussi qu'il y a là lieu d'ouvertures au pouvoir d'autorité, ce là, voilà ce qui occupe l'Ordre phallique.

Par ailleurs, au sujet du désir aliéné, il ne faut pas perdre de vue que la transgression du désir originaire n'affecte pas que les femmes. Vous aurez compris que des hommes sont sans cesse " mis au travail » pour assurer dominance au pouvoir (dit phallique par les psychanalystes), alors qu'Elle est mise au " travail de la réduction Unique " et elles sont dominées par les effets produits par cet appareil de pouvoir. Ces hommes mettent autant d'énergie à nier leurs désirs profonds pour « jouer " de pouvoir que certaines femmes mettent passion à désirer une fausseté amoureuse après laquelle elles sont amenées à soupirer.

Il ne s'agit donc pas ici d'hommes désaliénés en face d'une/ de femme/s aliénée/s, mais plutôt d'un mode de mise en place des pouvoirs qui transgresse l'aspiration profonde des individus sous prétexte d'un accomplissement (sous une forme jugée nécessaire . . . arbitrairement ?), de l'Ordre des choses, d'où il est dit qu'Une femme n'est pas toute en son essence, de là les femmes subissent les mêmes conditions.

12. Encore, p. 15 .

13. Encore, p. 13. 
INAPPROPRIÉE EN SON DÉSIR.

Paradoxe . . .

Dans notre proposition, nous avons glissé un double sens, laissant entendre qu'elle n'est pas toute parce qu'inappropriée en son désir ; nous avions aussi l'intention d'y laisser entendre qu'elle n'est pas toute inappropriée en son désir, voyons cela . . .

Elle, étrange chose être et ange, est-elle vivante en son désir en ce lieu " horsexe » ? Lacan nous dit qu'elle est mise au travail de l'Un ; "L'homme croit créer - il croit-croit-croit, il crée-crée-crée. Il crée-crée-crée la femme. En réalité, il la met au travail, et au travail de l'Un ${ }^{13 a}$.

Dans cette condition, se peut-il qu'elle soit sensible à tout ce qui apparaît de l'être avant d'être l'objet d'un néant métaphysique puis l'objet du rien dans la traduction husserlienne des lieux métaphysiques?

Est-elle susceptible de dé-celer quelque vérité fondamentale ? Car il s'agit bien ici, à n'en pas douter, du lieu des fondements du savoir métaphysique. À ce propos Lacan s'interroge ; «La question est en effet de savoir, dans ce qui constitue la jouissance féminine pour autant qu'elle n'est pas toute occupée de l'homme, et même dirais-je, que comme telle elle ne l'est pas du tout, la question est de savoir ce qu'il en est de son savoir ${ }^{14}$. C'est dire qu'il considère qu'elle n'est pas qu'une simple condition de vérité, il y aurait un " niveau où la femme, c'est la vérité $"{ }^{15}$.

CE DÉSIR, AILLEURS QUE DANS LE RAPPORT SEXUEL, RETENU.

Retenu, heureusement ! car «Ce n'est que de là où elle est toute, c'est-à-dire d'où la voit l'homme, rien que là que la chère femme peut avoir un inconscient ${ }^{16}$. Parce qu'Autre dans le corps parlant, là on s'en sert » à faire parler l'être parlant ici réduit à l'homme ${ }^{17}$.

13a. Encore, p. 13 .

14. Encore, p. 59.

15. Encore, p. 94.

16. Encore, p. 90. Nous comprenons ; peut avoir en son inconscient l'intuition de ce qui est.

17. Encore, p. 90. 
Ainsi elle ne cesse pas de s'écrire, par le fait qu'elle est " objet-porteur » d'un signifiant pur, par là inter-dite d'où elle est éventuellement sensible au signifiant, à son rapport au monde et aux choses. La femme, dit Lacan, "cherche un homme au titre de signifiant ${ }^{18}$. Le trouve-t-elle ? Ça, Lacan ne semble pas le savoir.

Ce qu'il sait c'est que . . . « si la femme n'était pas pas toute, si dans son corps, elle n'était pas pas toute comme être sexué de tout cela rien ne tiendrait ; bref l'Autre qui s'incarne ${ }^{19}$.

En somme cet Autre ne serait peut-être pas le m'être qu'il est ! Ici, quelle que soit la forme qu'on lui donne dans l'écriture ; m'être ou maître, il demeure l'indication d'un signifiant également considéré vrai dans l'appareil d'autorité, c'est-à-dire que le tenant se reconnaît empreint de l'être et/ou y est reconnu, ce qui lui vaut de devenir maître. Cet Autre n'est pas lui-même investi du signe, il serait plutôt traversé d'un signifiant alors qu'il n'est pas impossible que cette femme soit l'objet-source du signe.

L'ascétisme du maître, Lacan le conjugue ainsi ; " Je suis m'être, je progresse dans la m'êtrise, je suis m'être de moi comme de l'univers ", ici ajoute-t-il « l'univers c'est une fleur de rhétorique et le maître est le "con-vaincu " ${ }^{20}$. D'où nous considérons que La femme n'a pas de temporalité propre, elle ne peut qu'être la constante absoluité en leur système de renvoi. Le grand Autre ou Non-Être (principe de multiplicité chez Platon) ${ }^{21}$.

\section{EST L'OBJET D'UN GRAND NIA NIA.}

Nous sommes partie d'un «désir ailleurs que dans le rapport sexuel retenu> qui a pour cause une double négation opérée au lieu de «L/a femme ». Par le fait que celle-ci ne peut être reconnue comme femme, elle n'est retenue que pour une particularité, d'elle présente, à savoir qu'à l'ouvert situé d'elle apparaît à l'homme l'univers des discours. Ignorée, elle se trouve

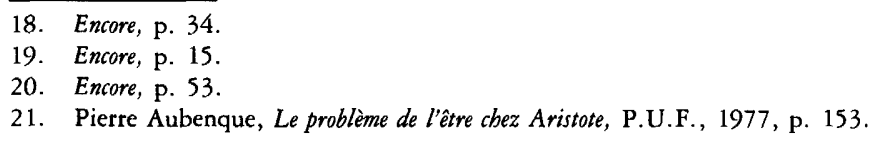


niée de tenants en tenants du signifiant. Cela Lacan l'écrit « nia nia ». Ce terme il l'écrit aussi «nyania ${ }^{22}$; de cette seconde formulation, nous déduisons l'existence d'un lieu où entre des hommes et la femme il n'y a pas négation (nya pas nia). Seraitce là où elle a rapport à « l'Un de la fusion universelle ${ }^{23}$, d'où la considération de vérité à elle rattachée ?

Quoi qu'il en soit, cela lui vaudra le risque de ce que ces messieurs nomment psychose et une certaine jouissance . . . «pas piquée des vers». . . que Lacan dit "au-delà du phallus " et qui dépasserait somme toute " celle qui se fait du coï ${ }^{24}$.

Quel peut être le ressort d'une telle jouissance sinon l'effet produit de l'Un de la fusion universelle en soi, en elle achevé, à partir d'elle soutenu, en quelque sorte pour cette femme investie des possibilités les plus grandes, le plus grand des effets se déroulant à son niveau au stade du miroir.

D'OÙ UNE FEMME RENCONTRE L'HOMME (L'homme) DANS LA PSYCHOSE.

Par le fait qu'elle est doublement niée s'exerce pour elle l'effet de la privation. Le jeu d'équilibre, opérationnel en toute position onto-théologique lui est ravi, cela lui vaut fantasmes, hystérie d'angoisse, apathie. Lacan a joué là-dessus, il appelle ça "l'amur " ${ }^{25}$. Nous interprétons ce terme comme désignant la réduction en un de l'amour et du mur. Cette femme est la source productive ; à la fois "l'âmoureuse » et l'emmurée de la fusion universelle. Elle est ce mur qui leur assure la circonscription de l'Espace, ainsi dans son corps se manifeste sous forme de fantasme la forclusion.

D'avoir à âmer « leur partenaire pourtant homo jusqu'à la garde, dont elles ne sortiront pas . . . ça ne peut en effet les conduire qu'à ce terme ultime (. . .) l'hystérie ${ }^{26}$. Ne se satisfaisant pas de cette constatation il entend mesurer la puissance de la psychose qui échoit à $\mathrm{L} / \mathrm{a}$ femme, à partir du champ freudien

22. “L'Étourdit ", p. 22.

23. Encore, p. 15 .

24. "L'Étourdit ", p. 23.

25. Encore, p. 11.

26. Encore, p. 79. 
recevoir les lieux qui du « Nom-du-Père s'élaborent en système de renvois ${ }^{27}$.

Par la suite, Lacan est amené à constater (est-ce en mesurant les champs du Nom-du-Père ?) qu'il n'y a pas de nom propre qui convienne au Père, il y découvre plutôt un jeu de rôle dont celui de "l'Homme masqué », jeu de cache-cache qui fait que toute réclamation faite au Nom-du-Père n'est que «Nom de Nom de Nom. Pas de Nom qui soit Nom-Propre, sinon le Nom comme ex-istence ${ }^{28}$.

De là nous pouvons déduire le sort réservé à $\mathrm{L} / \mathrm{a}$ femme ; elle ne peut être que traînée à la faveur des jeux de rôle de tout tenant de "Nom de Nom de Nom » y compris celui « d'Homme masqué » jusqu'à la condition psychotique. Il nous semble que Lacan a pu saisir là l'étendue de la Puissance.

N'entendant pas l'abandonner à son sort, il inaugure une autre mise en place ; «Le masque seul ex-isterait à la place de vide où je mets $L a$ femme. En quoi je ne dis pas qu'il n'y ait pas de femmes. La femme comme version du Père, ne se figurerait que de Père-version $"{ }^{29}$. Devons-nous entendre que ce n'est pas La femme qui témoignera de par sa mémoire mais plutôt l'ordre établi au Nom-du-Père qui se manifestera à partir de La femme? Personnellement, nous optons pour cette interprétation (plusieurs textes nous le confirment plus particulièrement celui intitulé L'Étourdit); là était le frayage lacanien ${ }^{30}$.

\section{AINSI LE CIEL QUI LUI ÉCHOIT LUI LAISSE JOUISSANCE « AU-DELÀ DU PHALLUS ".}

$L a$ femme a aussi une jouissance. L'espèce humaine est ainsi équilibrée, il n'est pas un lieu de projections qui ne soit susceptible de mener à l'exultation, que le champ universel du désir

27. "L'Étourdit ", p. 14. Ce texte est daté ; "Belœil, le 14 juillet 72 ".

28. Dans un recueil intitulé ; $A$ propos de l'éveil du printemps de Wedekind, Lacan signe une préface dans laquelle se trouvent des indications majeures sur ces intentions concernant La/les femme/s, p. 7 à 10, daté du 1 Septembre 1974, Christian Bourgois éditeur, 1974.

29. Ibid., p. 10, plus de deux ans après l'intention avouée de mesurer le Nom-du-Père comme lieu de privation. (voir note 27).

30. Par mémoire nous entendons toute inscription, trace . . présente dans l'organisme et/ ou l'organon, en aucun cas nous ne faisons allusion à ce que l'on pourrait confondre avec le souvenir. 
soit achevé ; à l'Imaginaire (sublimation), au corps sensible (orgasme) ou à la Raison (sérénité et/ou certitude) une jouissance prend forme. Personnellement nous considérons que cela fait partie des caractères innés de l'espèce humaine ${ }^{31}$. Quoi qu'il en soit elle a aussi jouissance, même si elle est exclue par l'ensemble des tenants de l'Ordre phallique à en "vivre " une. Lacan qualifie donc cette jouissance d' " au-delà du phallus » et dit " qu'il ne faudrait pas que ce soit celle-là » (qui soit) parce que cellelà précisément est de nature à la faire " pas toute ".

Malgré le fait qu'il suggère l'existence pour La femme d'une autre jouissance "sur laquelle la femme ne souffle mot" il semble convaincu qu'il " n'y en a pas d'autre que la jouissance phallique ${ }^{32}$.

Ce avec quoi nous sommes d'accord, nonobstant le fait qu'il en subsiste une autre qui, selon nous est de caractère inné, par le fait même échappe à la «domestication ».

Sur quoi repose cette jouissance ? nous avancions précédemment qu'il pouvait s'agir de l'effet produit en son stade du miroir, nous avançons maintenant que cela peut fort bien survenir d'un caractère inné propre à l'individu. L'un n'excluant pas l'autre.

Enfin quel est donc le statut de cette femme? «Comment savoir si, (. . .) le Père lui-même, (. . .) n'est que Nom entre autres de la Déesse blanche, celle (. . .) qui se perd dans la nuit des temps, à en être la Différente, l'Autre à jamais dans sa jouissance, - telles ces formes de l'infini dont nous ne commençons l'énumération qu'à savoir que c'est elle qui nous suspendra, nous ${ }^{33}$.

ALORS QUE LES CHAÎNES DE SES DÉSIRS, QUOIQUE AFFAIRE DE FÉMINITÉ, DEMEURENT LE LIEU DU MIGNON POUVOIR « D'OÙ ON LA DIT-FEMME ON LA DIFFAME ».

Il y a une traduction latine de la femme qui fait qu'elle ne peut être reconnue et respectée qu'en tant que mère. Là, à la femme on substitut la "féminité ». Et c'est précisément du lieu

31. Il serait intéressant ici s'approfondir les propositions de Konrad Lorenz; il nous rappelle entre autres qu'il y a " négligence complète de l'influence que la structure de l'individu, 
dit de la féminité que le "Mignon " institue sa présence au pouvoir à la place qui, dans d'autres conditions serait la sienne. Elle y est d'abord distinguée en tant que femme, voie à partir de laquelle on s'emploie à la diffamer.

Nous insistons sur le fait que la femme est accomplie d'une sommation de ses désirs qu'en aucun cas elle ne peut actualiser toute retenue qu'elle est à contenir l'Un.

Si d'aventure elle risquait de s'engager dans son propre champ de désir en vue de répondre à ces aspirations personnelles, immédiatement et de par la mise en place instituée dans l'Ordre phallique (traduction latine) s'applique la con-damnation : Elle répond à son désir de femme . . . elle est diffamée. Elle conserve ce désir en le portant au niveau abstrait . . . elle est refermée sur elle-même dans la dimension sacrée et demeure "Autre " à tout jamais. Dans de telles conditions peut-on prétendre qu'elle choisit délibérément d'être "Horsexe " ? Que c'est là son aspiration profonde?

Tout le champ du désir est occupé par «L'homme » dont certains se réclamant du "féminin " ${ }^{34}$. Lorsqu'il est question de L'homme, Lacan désigne ça du titre de "pourtouthomme », ce serait même de là qu'éventuellement elle aurait droit au discours, mais « les lieux de ce thommage se repèrent de faire sens du semblant ${ }^{35}$. D'où faux signifiant-m'être tout de même reconnu d'autorité, lieu duquel on peut prévoir les oppositions que $\mathrm{La}$ femme risque de présenter.

en tant que système organique, exerce sur la structure de la société qui l'englobe " de même qu'une "méconnaissance de la présence chez l'homme de types d'actions et de réactions innées caractéristiques de l'espèce. " Le comportement animal et bumain, Seuil/ Points, 1974.

32. Encore, p. 74.

33. L'éveil. . ., p. 10.

34. Dans son séminaire intitulé ; "La psychanalyse et l'énigme de la féminité " Claude Levesque insiste sur le fait que le féminin n'est pas la femme, nous pourrions même dire que jamais le dit féminin inclut $\mathrm{La}$ femme, d'où la nécessité formelle pour ceux se réclamant du féminin de la "barrer " quitte à prendre en file n'importe lesquelles autres. Ainsi s'opère l'Anéantissement de La femme et des femmes par la suite. De là comment s'étonner qu'avec le plus grand sérieux du monde certains mâles entreprennent des recherches prétendument pour nous éclairer sur ce qui dans le cerveau ou autres parties de l'organisme serait éventuellement en moins chez la femme et par là responsable de son caractère inférieur. Sacré pouvoir mignon!

35. "L'Étourdit ", p. 16. Ce " thommage " n'est pas sans allusion au jeu de l'hommage largement pratiqué dans le cercle fermé de l'élitisme mais il se peut aussi que Lacan y inclue une allusion au thomisme. Voir l'ensemble du texte. 
Étant elle-même porteuse du signifiant-m'être supposons, au pis-aller, qu'elle soit achevée et maintenue en constance qu'au seul niveau subliminal, selon nous elle ne saurait souscrire au faux semblant faisant autorité "pourtouthomme ». C'est autre chose que leur traduction qui la retiendrait et cette autre chose ne saurait que la mener en direction du signifiant-m'être . . . intuitivement.

Au mieux-aller, c'est-à-dire en la supposant consciente dans son savoir des enjeux dont elle est l'objet-de-réduction, elle ne saurait se plier à la loi du semblant sans offrir de sérieuses résistances.

De là l'impossibilité pour elle de s'inscrire, inconditionnellement, dans le discours d'autorité. Loi à laquelle tout mâle se plie.

Par ailleurs, d'autres tributaires du "féminin " (un lieu ayant fonction d'assurer la continuité du champ de désir de la femme mais auquel elle aura accès uniquement comme signifiantmêtre) s'assurent de la forclore. Là son désir à elle est supporté mais ... malheur de malheur, quoique son champ de désir bénéficie là d'une étendue, elle ne peut jamais y être qu'inversée. Pont de soupirs, s'il en est, où celui qui se réclame du féminin n'aimera jamais une personne de l'Autre sexe au point de la laisser être.

De La femme - suite au frayage lacanien - ne sauraiton pas espérer une désaliénation des femmes?

Du champ «freudo-lacanien " nous doutons que cela soit possible puisque Lacan lui-même nous dit que là où "ça parle " le rapport sexuel n'a pas lieu d'être ; "À cause de ce qu'elle parle, ladite jouissance, lui, le rapport sexuel, n'est pas ${ }^{36}$. En d'autres termes, là où le discours et ce de quoi il se soutient (un " ça parle " et/ou une phonè reconnue par une ou des institution/s) sont nantis d'effets de jouissance " ça " demeure le point d'attrait, d'où les participants de l'un et l'autre des sexes ne peuvent se rencontrer qu'en cet objet-tiers, conséquemment il n'y aura pas là de rapport sexuel, le copulatif étant le discours de l'inconscient et/ou celui d'autorité.

36. Encore, p. 57 . 
Or nous craignons, malgré le fait que Lacan met en place La femme en la délestant de la barre, qu'elle ne saura que dire plus sur ce qui la concerne. D'où même à partir du frayage lacanien La femme ne trouve pas délivrance. Elle risque plutôt d'être mise au travail . . . d'instruire le champ lacanien sur ce qui la concerne ; "ça parle " d'où l'impossibilité d'une levée de l'asservissement.

Ne risque-t-elle pas d'être mise au travail de nourrir le sujet?

D'où nous pouvons conclure qu'en de telles conditions La femme, même en possesśion de ces moyens, ne sait plus à quel homme sain se vouer (s'il en est !).

C'est qu'ils ont le pouvoir absolu d'actualiser là où Elle a celui « d'âmer » et d'y survivre par projections subliminales.

Ainsi va l'abnégation de La femme au Sacré et le Sacré à la trivialité des tenants de l'Ordre, soucieux de satisfaire l'aveugle désir de pouvoir qui les habite.

Le 28 Mai 1984

Département de philosophie Université de Montréal 